\title{
Prevalence of intestinal parasite, Shigella and Salmonella species among diarrheal children in Jimma health center, Jimma southwest Ethiopia: a cross sectional study
}

\author{
Getenet Beyene* and Haimanot Tasew
}

\begin{abstract}
Background: Diarrheal disease continues to be an important cause of morbidity and mortality among young children in developing countries including Ethiopia. Globally, intestinal parasite, Shigella and Salmonella species remain major contributors to acute enteric infections. The study was aimed at determining the frequency of intestinal parasite, Shigella and Salmonella species identified from diarrheic children at Jimma Health Centre, Jimma south west Ethiopia.
\end{abstract}

Methods: A health institution based cross sectional study was conducted from March to November 2012. A structured questionnaire was used for collection of data on socio- demographic characteristics. Parasite and bacteria identification as well as susceptibility testing was done using standard parasitological and bacteriological procedures.

Results: A total of 260 diarrheal children were included in the study. A total of 129 (49.6\%) samples were positive for intestinal parasite, Shigella and Salmonella species. Of these, 107 (41.1\%), 6 (2.3\%) and 16 (6.2\%) samples were positive for intestinal parasite, Shigella and Salmonella species respectively. The dominant isolated parasite was G. lamblia with prevalence of $13.5 \%$ followed by A. lumbricoides (11.5\%). The least identified parasites were Schistosoma mansoni and Taenia species accounting 0.4\% each. Multiple parasitic infections were observed in 19 (7.3\%) patients. Shigella species showed hundred percent resistances to ampicillin, amoxacillin, and cotrimoxazole. All Salmonella isolates were resistant against amoxicillin. All Shigella and Salmonella species were susceptible to ceftriaxone, ciprofloxacin and gentamycin.

Conclusion: The presence of reasonably high amount of intestinal parasite and Salmonella and Shigella species that are drug resistance to the commonly prescribed drugs is a treat to the children and community at large. Therefore, measures including health education, improvement of safe water supply, sanitation facilities and continuous monitoring of microbiological and antimicrobial surveillance is crucial.

Keywords: Intestinal parasite, Shigella, Salmonella, Susceptibility test, Jimma, Ethiopia

\footnotetext{
* Correspondence: regetene@yahoo.com

Department of Laboratory Sciences and Pathology, College of Public Health and Medical Sciences, Jimma University, Jimma, Ethiopia
} 


\section{Introduction}

Diarrheal disease continues to be an important cause of morbidity and mortality among young children in developing countries [1]. Children and young adults are the most affected, particularly in regions with limited resources and where hygienic measures are inadequate. Causes of diarrhea in endemic areas include a wide variety of bacteria, viruses, and parasites [2].

Gastrointestinal parasitic infections are amongst the most common infections worldwide. These cases are attributed to three common intestinal parasites: Ascaris lumbricoides, hookworm, and Trichuris trichiura. The global prevalence of parasitic diseases is estimated to be 478 million children for $A$. lumbricoides; 280 million for hookworms and 347 million children for T. trichiura [3]. In Ethiopia, intestinal parasitic infections are of serious public health concern [3]. According to a report by the Ministry of Health, helminthiasis is the third leading cause of outpatient visits in health institutions in 2005-2006 [4].

Recently, it was observed that the prevalence of intestinal parasitic infections in Jimma, Ethiopia [5] was as high as $86.2 \%$; whereas in Tigray, the overall prevalence was $48.1 \%$ [6]. According to the study findings conducted in Angolela, Ethiopia, on school children by Nguyen et al (2012), one-third of the children were infected with protozoan while $7.1 \%$ were found to have helminthic infections [7].

Among the diarrhoeal pathogens, Shigella continues to play a major role in etiology of inflammatory diarrhoea and dysentery, thus presenting a serious challenge to public-health authorities worldwide [8]. The few studies conducted on shigellosis in Ethiopia revealed that, Shigellosis and the emergence of antimicrobial resistant Shigella species is a major health problem [9-14]. Recent data different health institutions in Ethiopia have indicated that salmonellosis is a common problem and also showed the presence of a number of serogroups/ serotypes in humans, animals, food animals, food products animal origins and other food stuff [15-19].

Infections by most species of Shigella and Salmonella can be asymptomatic, or can be treated with rehydration solutions except for infection by invasive strains. The use of antibiotics might shorten the duration of diarrhea and limit the shedding of the organisms which otherwise might continue to spread among people and in to the environment and further pose a risk of transmission of infections. However, antimicrobial resistance is an overgrowing problem, and there is a need to monitor the susceptibility of common bacterial isolates to drugs used in the community to provide guidelines for the empirical treatment of bacterial infections.

In the present study, a prospective cross sectional study was conducted to determine the prevalence of intestine parasite, Shigella and Salmonella species among diahrroic children visiting Jimma Helath Center from March to November 2012, south West Ethiopia. We determined the prevalence of intestinal parasites, Shigella and Salmonella species in diarrhoeal stools of children including also the susceptibility to antimicrobial agents of the investigated pathogens.

\section{Material and methods}

\section{Study design, area and period}

A prospective cross sectional study was conducted to determine the prevalence of intestinal parasite, Shigella and Salmonella species among diarrheic children visiting Jimma Health Center, Jimma south West Ethiopia from March to November 2012.

\section{Sample size and sampling technique}

A total of 260 children were participated as a study subject. The sample size was determined based on the prevalence rate of the study done by Mache on children at Jimma [15] and calculated with the formula recommended by Daniel W [20].

\section{Demographic data collection}

Histories were taken from each child and informed consent was obtained from the parents or guardians before sample collection was attempted by the attending pediatrician. All relevant demographic, clinical and laboratory data were recorded and transferred to the questionnaire prepared for this study.

\section{Specimen collection and identification of pathogens}

Freshly passed stool and rectal swab was collected, placed immediately in Cary Blair transport medium (Oxoid Ltd, Basingstoke, UK) and transported to the laboratory within six hours of collection. For identification of Shigella and Salmonella species, specimens were placed in Selenite F enrichment broth (Oxoid) and incubated at $37^{\circ} \mathrm{C}$ for 24 hours, then subcultured onto deoxycholate agar (DCA) and xylose lysine deoxycholate agar (XLD) (Oxoid) agar at $37^{\circ} \mathrm{C}$ for $18-24$ hours. The growth of Salmonella and Shigella species was detected by their characteristic appearance on XLD agar (Shigella: red colonies, Salmonella red with a black centre) and DCA (Shigella: pale colonies, Salmonella black centre pale colonies). The suspected colonies were further tested through a series of biochemical tests to identify Shigella and Salmonella species [21].

\section{Parasitological examination of stool}

Stool specimens were obtained from all participants and examined for the presence of intestinal parasite cysts, eggs, trophozoites and larvae. In the laboratory, slides were prepared directly for wet mount in saline as well as in iodine and then were microscopically examined 
initially under low power (10X10 magnifications) bright field then under high-power (40X40 magnification) bright field. Finally the sample was concentrated using the procedure of formalin ethyl acetate technique 4 and iodine stained slides were prepared and examined microscopically.

\section{Susceptibility testing}

Antimicrobial drug susceptibility testing was carried out using disk diffusion method according to Clinical Laboratory Standards Institute (CLSI) guide lines [22]. The antibiotic discs used and their concentrations were:ceftriaxone (CRO, $30 \mu \mathrm{g})$, chloramphenicol (C, $30 \mu \mathrm{g})$, ciprofloxacin (CIP, $5 \mu \mathrm{g}$ ), gentamicin (GM, $10 \mu \mathrm{g}$ ), nalidixic acid (NA, $30 \mu \mathrm{g}$ ), trimethoprim-sulfamethoxazole/ cotrimoxazole (SXT, $25 \mu \mathrm{g}$ ) ampicillin (AMP $10 \mu \mathrm{g}$ ) and amoxicillin (AML, $20 \mu \mathrm{g}$ ). All antibiotic were obtained from Oxoid Limited, Basingstoke Hampshire, UK. A standard inoculum adjusted to $0.5 \mathrm{McF}$ arland was swabbed on to Muller- Hinton agar (Oxoid Ltd. Bashingstore Hampaire, UK); antibiotic disc were dispensed after drying the plate for 3-5 min and incubated at $37^{\circ} \mathrm{C}$ for 24 hours. The reference strains used as control were E.coli ATCC 25922.

Data were entered and analyzed using SPSS version 16.0 computer software. Ethical clearance was secured from Ethical Clearance Committee of College of Public Health and Medical Sciences Jimma University. Permission was obtained from Health center officials.

\section{Results}

Stool specimens of 260 children were collected and examined for the presence of intestinal parasites and cultured for Salmonella and Shigella species. Out of the total 260 study participants $114(43.8 \%)$ were males and 146 (56.2\%) were females showing an overall male to female ratio 1:0.8. The age of the studied children ranges from one month of age to 15 years with mean age of five year (+SD 4.1): the majority $(60.3 \%)$ of the study subjects were between 1-5 years of age and list frequency $(8.4 \%)$ was observed for children less than one years old (Figure 1).

Out of the 260 stool samples, 129 (49.6\%) samples were positive for intestinal parasite, Shigella and Salmonella species. Of these, 107 (41.1\%), 6 (2.3\%) and 16 (6.2\%) samples were positive for intestinal parasite, Shigella and Salmonella species respectively. The dominant isolated parasite was Giardia. lamblia with prevalence of $13.5 \%$ followed by $A$. lumbricoides (11.5\%). The least identified parasites were Schistosoma mansoni and Taenia species accounting $0.4 \%$ each (Table 1 ).

The distribution of enteropathogens according to the different age groups is listed in Table 2. The majority (58.1\%) of enteropathogens were found in children aged 1-5 years. Whereas, 26 (20.2\%), 21 (16.3\%) and seven (5.4\%) pathogens were observed in children within age groups of $6-10,11-15$ and less than one years, respectively. Multiple parasitic infections were observed in 19 patients (Table 3). Triple infection was observed in five children $(1.5 \%)$ and double parasitic infections were observed among 14 children. The commonest parasites in multiple infections were A. lumbricoides + G. lamblia. The commonest double infections were T. trichuria $+G$. lamblia + G. lamblia + E. histolytic. The commonest triple infections were $A$. lumbricoides $+H$. nana + G. lamblia (15.8\%). There was no confection of bacteria (Salmonella and Shigella species) and parasite among the children (Table 3).

Shigella species showed hundred percent resistances to ampicillin, amoxacillin, and cotrimoxazole while all (100\%) isolates were susceptible to ceftriaxone, ciprofloxacin and gentamicin (Table 4). Resistance to ampicillin,

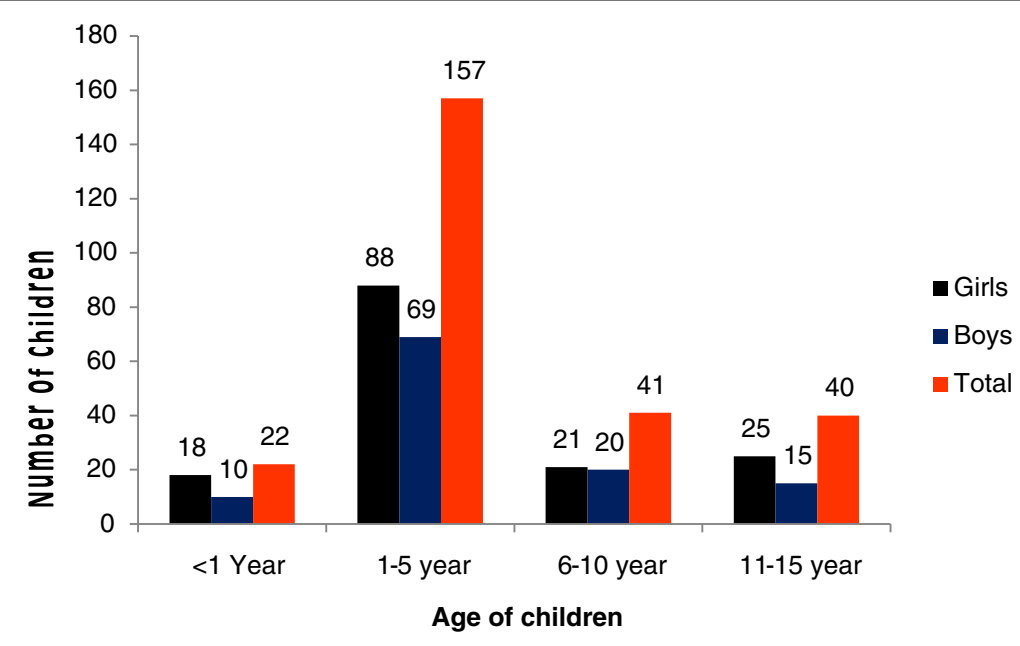

Figure 1 Age and sex distribution of the study group. 
Table 1 Frequency of intestinal parasite and bacteria isolated from 260 diarrheic children

\begin{tabular}{lc}
\hline Etiological pathogens & Patient $(\boldsymbol{n}=\mathbf{2 6 0})$ no. (\%) \\
\hline Paraite & $30(11.5)$ \\
A. lumbricoides & $8(3.1)$ \\
T. trichiura & $4(0.4)$ \\
Hook worm & $20(7.5)$ \\
H. nana & $1(0.4)$ \\
Taenia spp & $1(0.4)$ \\
S. mansoni & $35(13.5)$ \\
Giardia lamblia & $8(3.1)$ \\
E. histolytica & $\mathbf{1 0 7}(\mathbf{4 1 . 1 )}$ \\
Total & \\
Bacteria & $6(2.3)$ \\
Shigella species & $16(6.2)$ \\
Salmonella species & $\mathbf{2 2 ( 8 . 5 )}$ \\
Total & $\mathbf{1 2 9}(\mathbf{4 9 . 6 )}$ \\
Enteropahogens total &
\end{tabular}

cotrimoxazole, chloramphenicol and nalidixic acid was observed in 62.5, 31.3, 18.8\% and 12.5 of Salmonella isolate respectively. All Salmonella isolates were resistant against Amoxicillin and susceptible to ceftriaxone, ciprofloxacin and gentamicin (Table 4).

Over all three resistance patterns were seen among Shigella isolates. All Shigella species were multi-drug resistant (resistant to three or more antimicrobial drugs). About $66.6 \%$ of Shigella species were resistant to three (ampicillin, amoxacillin, cotrimoxazole) antibiotics. On

Table 2 Age distribution of intestinal parasite, Shigella and Salmonella species among the study participants

\begin{tabular}{|c|c|c|c|c|}
\hline & No. (\%) & & & \\
\hline Age group in years & $<1$ & $1-5$ & $6-10$ & $11-15$ \\
\hline \multicolumn{5}{|l|}{ Parasite } \\
\hline A. lumbricoides & - & $13(10.1)$ & $10(7.8)$ & $7(5.4)$ \\
\hline T. trichiura & - & $5(3.9)$ & $1(0.8)$ & $2(1.6)$ \\
\hline Hook worm & - & $1(0.8)$ & $1(0.8)$ & $2(1.6)$ \\
\hline H. nana & - & $14(10.9)$ & $4(3.1)$ & $2(1.6)$ \\
\hline Taenia spp & - & - & $1(0.8)$ & - \\
\hline S. mansoni & - & - & $1(0.8)$ & - \\
\hline Giardia lamblilia & - & $28(21.7)$ & $3(2.3)$ & $4(3.1)$ \\
\hline E. histolytica & $1(0.8)$ & $3(2.3)$ & $1(0.8)$ & $3(2.3)$ \\
\hline \multicolumn{5}{|l|}{ Bacteria } \\
\hline Shigella species & $1(0.8)$ & $4(3.1)$ & $1(0.8)$ & - \\
\hline Salmonella species & $5(3.9)$ & $7(5.4)$ & $3(2.3)$ & $1(0.8)$ \\
\hline Total & $7(5.4)$ & $75(58.1)$ & $26(20.2)$ & $21(16.3)$ \\
\hline
\end{tabular}

Table 3 Frequency of multiple (double and triple) parasitic infections of children

\begin{tabular}{ll}
\hline Parasite combinations & No \% \\
\hline As, Hy, GL & $3(15.8)$ \\
As, Tt, GL & $1(5.3)$ \\
As, Tt, EH & $1(5.3)$ \\
As, HW & $2(10.5)$ \\
As, Tt & $2(10.5)$ \\
As, GL & $2(10.5)$ \\
Hy, GL & $2(10.5)$ \\
Tt, GL & $3(15.8)$ \\
GL, Eh & $3(15.8)$ \\
Total & $19(100.0)$ \\
\hline
\end{tabular}

Keys: - Al- Ascaris lumbricoide, Hy: Hyminolopis nana, GL: Giardia lamblia, Tt: Trichuris trichiuria, Eh: Entamoeba histolytica, HW: Hook worm.

the other hand $62.5 \%$ of Salmonella species were multidrug resistant, ranging from 2 to 4 drugs (Table 5).

\section{Discussion}

The study results indicated, of the total 260 study participants, 107 (41.1\%) of the symptomatic children were infected with one or more intestinal parasites. It is comparable with study done by Unasho in southern Ethiopia, where 170 (41.9\%) children were found to have single and double intestinal parasitic infections [23], but higher than study conducted in Gondar where the observed prevalence of intestinal parasites was 34.2\% [24] and the study conducted in Gamo area where 342 (39.9\%) study subjects were found positive for at least one intestinal parasite [25]. Our study prevalence result was lower compared with reports of other similar studies, $72.9 \%$ in Gondar, Azezo [25], 83\% in Jimma [5] and 83.8\% in South East of Lake Langano [26]. These variations in prevalence might be due to differences in climatic conditions, environmental sanitation, economic and educational status of parents and study subjects, and previous control efforts. The low prevalence of intestinal parasite in this study compared to

Table 4 Resistance pattern of Shigella and Salmonella species

\begin{tabular}{lll}
\hline Antimicrobial & \multicolumn{2}{l}{ No $(\%)$ of resistance } \\
\cline { 2 - 3 } & Shigella species $(\mathbf{n}=\mathbf{6})$ & Salmonella spices $(\mathbf{n}=\mathbf{1 6})$ \\
\hline Ampicillin & $6(100)$ & $10(62.5)$ \\
Amoxicillin & $6(100)$ & $16(100)$ \\
Cotrimethoxazole & $6(100)$ & $5(31.3)$ \\
Nalidixic acid & $1(16.7)$ & $2(12.5)$ \\
Ceftriaxone & - & - \\
Ciprofloxacin & - & - \\
Chloramphenicol & $1(16.7)$ & $3(18.8)$ \\
Gentamicin & - & - \\
\hline
\end{tabular}


Table 5 Antibiogram of Shigella and Salmonella isolates

\begin{tabular}{lcc}
\hline Resistance pattern & \multicolumn{2}{c}{ Resistant isolates no. (\%) } \\
\cline { 2 - 3 } & $\begin{array}{c}\text { Shigella species } \\
(\mathbf{N}=\mathbf{6})\end{array}$ & $\begin{array}{c}\text { Salmonella species } \\
(\mathbf{N}=16)\end{array}$ \\
\hline Amp & - & $1(6.3)$ \\
Amx & - & $5(31.3)$ \\
Amp, Amx & - & $1(6.3)$ \\
Amp, Amx, C & - & $1(6.3)$ \\
Amp, Amx, Sxt & $4(66.6)$ & $3(18.8)$ \\
Amp, Amx, NA, & - & $2(12.5)$ \\
Amp, Amx, Sxt, C & $1(16.7)$ & $2(12.5)$ \\
Amp, Amx, Sxt, NA, & $1(16.7)$ & - \\
\hline
\end{tabular}

Keys:- Amp: Ampicillin, Amx: Amoxicillin, C: Chloramphenicol, NA: Nalidixic acid, Sxt: Cotrimethoxazole.

the other previous studies in Jimma (5) and elsewhere in the country (7) could be due to increased awareness of the community about personal and environmental hygiene from the continuous awareness creation and interventions made by the health science students from Jimma University during their practical training conducted in the field as well as in different health institutions.

Among helminthiasis, Ascaris lumbricoid (11.5\%) was the most prevalent parasite and followed by $H$. nana and T.trichiura, whereas giardiasis was the leading infection among protozoan infections. Though the rate of prevalence is different the dominancy of $A$. lumbricoides is in line with the study result by Abayne in south Ethiopia [23] and with previous studies done in Assedabo (Jimma, Ethiopia), where Ascaris lumbricoides was the leading (56.4\%) [5]. But different with study conducted in Gondar [24] where the dominant parasite was H. nana. The difference could be mainly difference in climatic condition.

Many studies had already demonstrated the high prevalence of parasitic infections among Ethiopian children in various parts of the country [5,27-29]. In this study, A. lumbricoides (3.5\%) and T. trichiura (3.1\%) were found to be the dominant soil transmitted helminthes. Studies done by Ali et al. (1999) and Roma and Solomon (1997) reported higher rates of A. lumbricoides (54.6\% vs. $75.2 \%)$ and T. trichiura (21\% vs. $24.4 \%)$ respectively [5,29]. Our study data showed comparable prevalence rate with reported by Tadesse (2005) where the prevalence rate of $A$. lumbricoides and T. trichiura were $3.6 \%$ and $3.9 \%$, respectively [30]. A prevalence rate of $3 \%$ for $H$. nana is higher than earlier findings, which were $1.1 \%$ in Wondogenet, Ethiopia [23], 1.3\% in south Wollo [31] and lower than with the findings of Tadesse, (2005) and Haileamlak (2005) with frequency rate of $10.1 \%$ and $4.3 \%$ respectively $[27,30]$.

Except in the early stage/age (where mostly rely on breast feeding), parasitic infections were decreasing as the age of the children increases, which could be due to acquired immunity as they are exposed more and more to infections as well as developing awarness to personal hygiene. In this study multiple parasitic infections were seen in 19 children (7.3\%) from the total 260 study subjects and $17.8 \%$ among children who were infected with parasites and this result is higher than the study conducted in Gondar, where multiple infections (polyparasitism) occurred in 14 individuals or $4.6 \%$ of the total examined subjects and $13.5 \%$ of those who had intestinal parasites [32]. The difference could be due to geographical location or variation in study subject sample size.

Comparison with previous study results conducted in different parts of Ethiopia is difficult since the parasite prevalence varies with agro-ecozone, altitude and other environmental factors which are not studied here. Shigellosis is primary a pediatric disease, with more than half of all infections occurring in children between six month to 10 years of age as observed in previous Ethiopian study [14]. The isolation of Shigella species (2.3\%) in this study is lower than (5\%) reported by Mache, 2001 [14] (20.1\%) from the same study subjects and area.

Even though, the study was conducted in different age groups, our prevalence rate of $2.3 \%$ lowers than that a report by Ashenafi, 1983 (9\%) [33] and 11.7\% isolation rate reported by Asrat et al. 1999 at Tikur Anbessa, Ethio-Swedish children's hospital [34], a report by Ayalu (6.7\%) in Harar [35] and a report $15.6 \%$ by Hiruy in Gondar [36]. The low isolation of Shigella in this study compared to the previous study in Jimma (14) could be due to increased awareness of the community about personal and environmental hygiene from the continuous interventions made by the health science students form Jimma University during their filed practice.

Epidemiological investigation of salmonellosis in developing countries like Ethiopia is difficult because of the very limited scope of the studies and lack of coordinated surveillance systems. The overall prevalence of Salmonella in this study was $6.2 \%$. This is comparable with studies conducted in Ethiopia at different times, $4.5 \%$ in Addis Ababa [37], 6.4\% in Addis Ababa [16], 4.5\% in Addis Ababa [33] and higher than the findings reported by Asrat et al. 1999 (3.8\%) in Addis Ababa [34] but lower than reported in Jimma (15\%) [15]. Antibiotic susceptibility data to Shigella isolates showed that all isolates were resistance to ampicillin, amoxacillin, and cotrimoxazole. Similarly a study conducted in Awassa showed that all isolates were resistant to amoxacillin and ampicillin (12). The development of high resistance of Shigella species against the commonly used antibiotics was witnessed by other investigators in different periods. In Hawassa high rate of resistance of Shigella species to ampicillin (93\%), erythromycin (90\%), tetracycline (90\%) and cotrimoxazole (56\%) was reported by Roma et al [12], in Gondar, high 
antibiotic resistance was documented against ampicillin (79.9\%), tetracycline $(86 \%)$, and cotrimoxazole $(73.4 \%)$ by Yismaw et al. [38]. Asrat reported isolation of Shigella species with high resistance to erythromycin (100\%), Tetracycline (97.3\%), and ampicillin (78.7\%) in Addis Ababa [39]. High resistance against amoxicillin (100\%) and ampicillin (100\%) was also reported by Reda et al. in Harar [35]. Our Shigella isolates were highly susceptible to ceftriaxone, ciprofloxacin and gentamicin. Comparatively high rate of resistance to ciprofloxacin (8.3\%) was reported in Gondar. In parallel to our result lower (2\%) resistance rate was reported from Gondar [38]. Comparable to the study conducted by Daniel [39] in Addis Ababa where all Shigella isolates were susceptible to gentamicin. The low resistant rate of isolates to chloramphenicol (18.8\%), could be that physicians stopped to prescribe the drug before long time a go and once again the strains started to become sensitive. All Shigella isolates were MDR (resistant to two or more drugs). Similar findings were seen in other studies in Ethiopia [12,38].

Infection with non-typhoidal Salmonella in infants and children commonly produces self-limited diarrhoea. Studies have indicated that antimicrobial treatment for uncomplicated gastroenteritis does not shorten the duration and severity of symptoms; in contrast, it may prolong fecal excretion, increase the risk of relapse, and result in the emergence of antibiotic resistance [40]. Nevertheless, if extra-intestinal complications occur, effective antimicrobial treatment is essential. Multidrug resistant phenotypes have been increasingly described among Salmonella species worldwide, according to the infectious disease report released by the WHO in 2000 [41].

In this study, Salmonella isolates showed high re resistant to ampicillin and amoxicillin which is comparable with previous study done in Harar, Ethiopia where the highest level of resistance was detected to ampicillin (100\%) and amoxicillin (100\%) [35]. a relatively similar pattern of resistance (74 to $97.3 \%$ ) was reported from other parts of the country [42] and outside [43]. Our study findings showed that all Salmonella species were susceptible to ceftriaxone and ciprofloxacin, which is inline with recently study results conducted in Nigeria where all isolates were susceptible to ciprofloxacin, and ceftriaxone [44]. Un like most previous study findings, in this study Shigella and Salmonella species showed low resistance level to chloramphenicol, this could be due abandoning of prescribing the drug by the responsible health personnel before a long time ago.

\section{Conclusion}

This study indicated that intestinal parasite and some enteric bacteria such as Salmonella and Shigella species are responsible for the majority cases of diarrhoel in children. The results of the present study suggested that antibiotics which were more commonly prescribed in the study area previously, like ampicillin, amoxicillin and cotrimethoxazole developed resistance to Shigella and Salmonella species and should not be used as empirical treatment of diarrhoea in children at least in the study area. Therefore, measures including health education, improvement of safe water supply, sanitation facilities and continuous monitoring of microbiological and antimicrobial surveillance is crucial.

\section{Competing interests}

The authors declared that they have no competing interests.

\section{Authors' contribution}

GB: Participated from inception of the research question to design, analysis, interpretation and preparation of the manuscript. HT: - Participated in proposal development, analyzed the data, edit and wrote the manuscript for publication. Both authors have given final approval of the version to be published.

\section{Acknowledgements}

We would like to acknowledge study participants or their guardians who were volunteers to participate in the study. We are grateful to acknowledge Dr. Rene S. Hendriksen from Technical University of Denmark, National Food Institute, for giving constructive comment during preparation of the manuscript. We would like to acknowledge Jimma University for funding the research work and the Ethiopian Health and Nutrition Research Institute (EHNRI) for providing us control organisms.

\section{Received: 2 December 2013 Accepted: 1 February 2014}

Published: 5 February 2014

\section{References}

1. Bern C, Martines J, Zoysa I, Glass Rl: The magnitude of the global problem of diarrheal disease: a ten-year update. Bull. WHO 1992, 70:705-714.

2. Obi $C L$, lgumbor EO, Bessong PO, Guerrant SR: Prevalence of intestinal parasitic and bacterial pathogens in diarrhoeal and Non-diarroeal human stools from Vhembe district, South Africa. J Health Popul Nutr 2009, 27(6):739-745

3. Bethony J, Brooker S, Albonico M: Soil-transmitted helminth infections: ascariasis, trichuriasis, and hookworm. Lancet 2006, 367:1521-1532.

4. Ministry of Health $(\mathrm{MOH})$ : Health and Health Related Indicators. Addis Ababa, Ethiopia: Planning and Programming Department; 2011.

5. Ali I, Mekete G, Wodajo N: Intestinal parasitism and related risk factors among students of Asendabo Elementary and Junior Secondary school, South Western Ethiopia. Ethiop J of Health Dev 1999, 13:157-161.

6. Asfaw ST, Giotom L: Malnutrition and enteric parasitosis among under-five children in Aynalem Village, Tigray. Ethiop J of Health Dev 2000, 14:67-75.

7. Nguyen NL, Gelaye B, Aboset N, Kumie A, Williams MA, Berhane Y: Intestinal parasitic infection and nutritional status among school children in Angolela, Ethiopia. J prev med hyg 2012, 53:157-164.

8. Kansakar P, Malla S, Ghimire RG: Shigella isolates of Nepal: Changes in the incidence of Shigella subgroups and trends of antimicrobial susceptibility pattern. KU Med J 2007, 5:32-35.

9. Geyid A: Shigellosis in Ethiopia: review of studies. Ethio J Biol Sci 2004, 3:91-192.

10. Afeworki G, Lirneneh Y: Multiple drug resistance within Shigella serogroups. Ethiop Med J 1980, 18:7-11.

11. Senait K, Abera G, Sileshi L, KidaneMariam M: Clinical profile and drug susceptibility pattern of strains isolated from children in Addis Ababa. Ethiop Med J 1993, 37:19-27.

12. Roma B, Worku S, T/Mariam S, Langeland N: Antimicrobial susceptibility pattern of Shigella isolates in Awassa. Ethiop J of Health Dev 2000, 14:154

13. Tiruneh M: Serodiversity and antimicrobial resistance pattern on Shigella isolates at Godar University Teaching Hospital, North West Ethiopia. Jpn J Infect Dis 2009, 62:93-97.

14. Mache A: Antimicrobial resistance and serogroups of Shigella among paediatric outpatients in southwest Ethiopia. East Afr Med J 2001, 78:296-299 
15. Mache A: Salmonella serogroup and their antibiotic resistance patterns isolated from diarrhoeal stools of pediatric out patients in Jimma Hospital and Jimma Health Center, South West Ethiopia. Ethiop J Health Sci 2002, 37:37-45.

16. Mache A, Mengistu Y, Cowley S: Salmonella serogroups identified from adult diarrhoeal out-patients in Addis Ababa, Ethiopia: antibiotic resistance and plasmid profile analysis. East Afr Med J 1997, 74:183-186.

17. Molla B, Mesfin A, Alemayehu D: Multiple antimicrobial resistant Salmonella serotype isolated from chicken carcass and giblets in Debrezeit and Addis Ababa, Ethiopia. Ethiop J Health Dev 2003. 17:131-149.

18. Beyene G, Asrat D, Mengistu Y, Aseffa A, Wain J: Typhoid fever in Ethiopia. $J$ Infect Dev Ctries 2008, 2:448-453.

19. Beyene B, Nair S, Asrat D, Mengistu Y, Engers H, Wain J: Multidrug resistant Salmonella Concord is a major cause of salmonellosis in children in Ethiopia. J Infect Dev Ctries 2011, 5(1):023-033.

20. Daniel W: Biostatictics: A foundation for analysis in the Health Sciences. USA: John Wiley abd Sons; 1983

21. Forbes BA, Sahm DF, Weissfeld AS: editors. Bailey \& Scott's diagnostic Microbiology. 11th edition. Philadelphia: Mosby Co; 2002:368-375. and 204-13 22.

22. National Committee for Clinical Laboratory Standards: Performance standard for antimicrobial disk susceptibility tests: approved standards. M2-A7. PA, USA: NCCL, Villanova; 2000.

23. Unasho A: An Investigation of Intestinal Parasitic Infections among the Asymptomatic Children in, Southern Ethiopia. Int J Child Helath Nutr 2013, 2:212-222.

24. Endris M, Lemma W, Belyhun Y, Moges B, Gelaw A, Angaw B: Prevalence of intestinal parasites and associated risk factors among students of Atse Fasil general elementary school Azezo, Northwestern Ethiopia. Ethiop J Health Biomed Sci 2010, 3(1):25-33.

25. Wegayehu T, Tsalla T, Seifu B, Teklu T: Prevalence of intestinal parasitic infections among highland and lowland dwellers in Gamo area, South Ethiopia. BMC Public Health 2013, 13:151.

26. Legesse M, Erko B: Prevalence of intestinal parasites among school children in rural area close to the south east of Lake Langano, Ethiopia. Ethiop J Health Dev 2004, 18(2):116-120.

27. Hileamlak A: Intestinal parasite in asymptomatic children in South West Ethiopia. Ethiop J Health Sci 2005, 15:108-118.

28. Merid Y, Hegazy M, Mekete G, Teklemariam S: Intestinal helminthic infections among children at lake Awassa area, south Ethiopia. Ethio J Health Dev 2001, 15(1):31-37.

29. Roma B, Worku S: Magnitude of Schistosoma mansoni and intestinal infections among school children in Wondo-Genet zuria, Southern Ethiopia. Ethiop J Health Dev 1997, 11:125-129.

30. Tadesse $\mathrm{G}$ : The prevalence of intestinal helminthic infections and associated risk factors among school children in Babile town, Eastern Ethiopa. Ethiop J Health Dev 2005, 19:140-147.

31. Assefa T, Woldemeichea T, Dejene A: Intestinal parasitism among students in three localities in south Wello, Ethiopia. Ethiop J Health Dev 1998, 12:231-235.

32. Gelaw A, Anagaw B, Nigussie B: Prevalence of intestinal parasitic infections and risk factors among schoolchildren at the University of Gondar Community School Northwest Ethiopia: a cross-sectional study. BMC Public Health 2013, 13:304

33. Ashenafi M: The prevalence of Salmonella, Shigella and Yersinia enterocolitica in adult diarrhoea out-patients in some hospital of Addis Ababa. Addis Ababa: M.Sc thesis School of graduate studies; 1998

34. Asrat D, Hathaway A, Ekwall E: Studies on enteric campylobacteriosis in Tikur Anbessa and Ethio-Swedish children's hospital, Addis Ababa, Ethiopia. Ethiop Med J 1999, 37:71-84.

35. Reda A, Seyoum B, Yimam J, Andualem G, Fiseha S, Vandeweerd JM: Antibiotic susceptibility patterns of Salmonella and Shigella isolates in Harar, Eastern Ethiopia. J Infect Dis Immun 2011, 3(8):134-139.

36. Huruy K, Kassu A, Mulu A, Worku N, Fetene T, Biadglegne S, Belyhun Y, Muche A, Gelaw A, Anagaw B, Yifru S, Wondie Y, Bekele A, Tiruneh M, Reissig D, Moges F: Intestinal Parasitosis and Shigellosis among Diarrheal Patients in Gondar Teaching Hospital, Northwest Ethiopia. BMC Research Notes 2011, 4:472

37. Ashenafi M, Gedebou M: Salmonella and Shigella in adult diarrhoea in Addis Ababa-prevalence and antibiograms. Trans $R$ Soc Trop Med Hyg 1985, 79:719-721.
38. Yismaw $G$, Negeri C, Kassu A: A five-year antimicrobial resistance pattern observed in Shigella species isolated from stool samples in Gondar University Hospital, northwest Ethiopia. Ethiop J of Health Dev 2006, 20(3):194-198.

39. Asrat D: Shigella and Salmonella serogroups and their antibiotic susceptibility patterns in Ethiopia. East Mediterr Health J 2008, 14(4):760-767

40. Chiu CH, Lin TY, Ou JT: A clinical trial comparing oral azithromycin cefixime and no antibiotics in the treatment of acute uncomplicated Salmonella enteritis in children. J Paediatr Child Health 1999, 35:372-374.

41. WHO: Overcoming antibiotic resistance. In World Health Organisation Report in Infectious Disease. Geneva: World Health Organisation; 2000.

42. Aseffa A, Gedlu E, Asmelash T: Antibiotic resistance of prevalent Salmonella and Shigella strains in northwest Ethiopia. East Afr Med J 1997, 74:708-13.

43. Karuiki S, Hart CA: Global aspects of antimicrobial-resistant enteric bacteria. Curr Opin Infect Dis 2001, 14:579-586.

44. Cajetan I, Bassey B, Florence IK, Nnennaya1 IR, Casmir AA: Salmonella species associated with childhood acute gastroenteritis in federal capital territory Abuja. Nigeria. British Microbiol Res J 2013, 3(3):431-439.

\section{doi:10.1186/1476-0711-13-10}

Cite this article as: Beyene and Tasew: Prevalence of intestinal parasite Shigella and Salmonella species among diarrheal children in Jimma health center, Jimma southwest Ethiopia: a cross sectional study. Annals of Clinical Microbiology and Antimicrobials 2014 13:10.

\section{Submit your next manuscript to BioMed Central and take full advantage of:}

- Convenient online submission

- Thorough peer review

- No space constraints or color figure charges

- Immediate publication on acceptance

- Inclusion in PubMed, CAS, Scopus and Google Scholar

- Research which is freely available for redistribution 